\title{
Use of cell phones and brain tumors: a true association?
}

\author{
Ettore Beghi ${ }^{1}$
}

Published online: 11 May 2017

(C) Springer-Verlag Italia 2017

The association between use of mobile cell phones and brain tumors has long been debated in the scientific literature and even in the lay press. The underlying biological mechanism is represented by the adverse effects of radio-frequency electromagnetic fields emitted by mobile phones, categorized as possible human carcinogens by the WHO International Agency for Research on Cancer [1]. Investigations of the effects produced by radio frequencies revealed increased risk of cell death and cancer development in experimental animals [2, 3]. Pathophysiological findings include calcium efflux from cell membranes, increased expression of stress proteins, influence on channels/gap junctions in cell membrane, overproduction of reactive oxygen species, ornithine decarboxylase activation, reduction in melatonin levels, decrease in protein kinase $\mathrm{C}$ activity, damage to DNA and change in gene expression in brain cells, and altered blood-brain barrier [4]. Electromagnetic fields have been implicated in several clinical conditions that include childhood leukemia, brain tumors, genotoxic effects, neurological effects and neurodegenerative diseases, immune system deregulation, allergic and inflammatory responses, breast cancer, miscarriage, and cardiovascular effects [5].

In this issue, Dr. Manya Prasad and co-workers performed a systematic review and a meta-analysis of published reports and came to the conclusion that prolonged use of cell phones is associated with a moderately high but statistically significant risk of brain tumors [6]. The conclusion is supported by the consistency of the results of original reports (most of them being non-significant simply because the study was

Ettore Beghi

ettore.beghi@marionegri.it

1 Laboratorio di Malattie Neurologiche, IRCCS-Istituto di Ricerche Farmacologiche Mario Negri, Via Giuseppe La Masa 19, 20156 Milan, Italy underpowered) and by a biological gradient (significance was most frequent after prolonged exposure). The authors concluded that the contrasting findings can be explained by the differing quality of published reports and by the source of funding, low-quality and industry-funded studies having failed to detect any association. The study findings have been confirmed by another recent meta-analysis that included 11 studies and showed that there was no overall association between mobile phone use and gliomas but found a slight but significant association when exposure was more than 5 years [7].

The issue addressed by Dr. Prasad has relevant safety implications in consideration of the increasing number of cell phone users in the world. For this reason, the medical community should pay special attention to these results. However, a note of caution should be raised, in light of the weight of the available evidence, to prevent any unjustified alarm to the general public. First of all, the positive results of some casecontrol studies have not been confirmed by cohort studies. Case-control studies, even when methodologically robust, cannot replace cohort studies in confirming or disproving an association between a given exposure and a disease. Cohort studies, unlike case-control studies, are characterized by a predefined definition of the type, length, and intensity of exposure and by an active follow-up in search of incident cases of the disease of interest. In this context, none of the cohort studies found an increased risk of brain tumors in people exposed to cell phones. Second, the increased risk of brain tumors in case-control studies, if proven, is at best modest and, as brain tumors are rare diseases, the total number of tumors appears only slightly increased. Third, the temporal association between exposure and disease onset cannot be confirmed. The biological onset of tumors' growth (including brain tumors) is unknown and the possibility that at the time of exposure the tumor was already present in the brain cannot be excluded. Fourth, a clear dose-response effect has never been 
confirmed. Last, electromagnetic fields have been implicated in neurodegenerative disorders but the experimental findings are controversial and, except for Alzheimer disease, epidemiological evidence supporting a true association has never been provided [8].

My personal skepticism is only motivated by the lack of robust evidence of association between use of cell phones and brain tumors. However, absence of evidence does not necessarily mean that an association is inexistent. A reasonable suspicion of risk exists based on evidence of biological effects at environmentally relevant levels that, with prolonged exposures, may be presumed to impact on population health. The prolonged use of cell phones should thus be actively monitored and further studies are awaited to provide definite findings to confirm or disprove this purported link.

\section{Compliance with ethical standards}

Conflict of interest E. Beghi reports grants from UCB-Pharma, grants from Shire, grants from EISAI, personal fees from Viropharma, grants from the Italian Ministry of Health, grants from the European Union, grants from Fondazione Borgonovo, and grants from Associazione IDIC 15, outside the submitted work.

\section{References}

1. Baan R et al (2011) Carcinogenicity of radiofrequency electromagnetic fields. Lancet Oncol 12(7):624-626

2. Tillmann $T$ et al (2010) Indication of cocarcinogenic potential of chronic UMTS-modulated radiofrequency exposure in an ethylnitrosourea mouse model. Int J Radiat Biol 86:529-541

3. Lerchl A et al (2015) Tumor promotion by exposure to radiofrequency electromagnetic fields below exposure limits for humans. Biochem Biophys Res Commun 459(4):585-590

4. Sri KN (2015) Mobile phone radiation: physiological \& pathophysiologcal considerations. Indian J Physiol Pharmacol 59(2):125-135

5. Hardell L, Sage C (2008) Biological effects from electromagnetic field exposure and public exposure standards. Biomed Pharmacother 62(2):104-109

6. Prasad M, Kathuria P, Nair P, Kumar A, Prasad K (2017) Mobile phone use and risk of brain tumours: a systematic review of association between study quality, source of funding, and research outcomes. Neurol Sci DOI. doi:10.1007/s10072-017-2850-8

7. Wang Y, Guo X (2016) Meta-analysis of association between mobile phone use and glioma risk. J Cancer Res Ther 12(Suppl):C298$\mathrm{C} 300$

8. Consales C, Merla C, Marino C, Benassi B (2012) Electromagnetic fields, oxidative stress, and neurodegeneration. Int J Cell Biol 2012: 683897 